\title{
Das chinesische Selbst \\ Die psychoanalytisch orientierte Psychotherapie in der \\ Volksrepublik China ${ }^{1}$
}

Antje Haag (Hamburg)

Zusammenfassung: Vor dem Hintergrund ihrer 20-jährigen Erfahrung als Dozentin für psychoanalytisch orientierte Psychotherapie in der Volksrepublik China diskutiert die Autorin Fragen, die im Zusammenhang mit dem Transfer einer am Individuum orientierten Therapiemethode in ein Land mit einer kollektivistisch-konfuzianischen Tradition gestellt werden können. Zunächst werden die kulturbedingt unterschiedlichen Konstruktionen des Selbst beschrieben, die einen besonderen Kommunikationsstil und - im Fall Chinas - eine ausgeprägtere Schamanfälligkeit implizieren. Psychoanalytische Grundannahmen wie Abstinenz, therapeutische Ich-Spaltung, Introspektionsfähigkeit und Übertragung können, ebenso wie das traditionell konfuzianische Harmoniestreben, aufkulturell immanente Probleme treffen. Eine spezifische Abwehrformation, die «Ah-QMentalität», wird vorgestellt und im Sinne eines daoistisch fundierten Denkens verstanden. Schliesslich wird diskutiert, ob bei psychoanalytisch orientierter Behandlungstechnik in weniger individualisierten Kulturen Modifikationen sinnvoll sind.

Schlüsselwörter: kulturspezifische Besonderheiten, das Selbst in kollektivistischen Gesellschaften, Psychoanalyse in China

\section{Vorspann}

Als Vorspann soll ein kleiner Exkurs in die Annalen europäisch-chinesischen Wissenschafts- und Kulturaustauschs dienen. Der erste fruchtbare interkulturelle Dialog begann vor etwa 400 Jahren durch die jesuitischen China-Missionare. In Deutschland war es Leibniz (2006), der schon früh der chinesischen Kultur und insbesondere ihren wissenschaftlichen Errungenschaften seine Aufmerksamkeit geschenkt hatte. Er sammelte und verarbeitete fasziniert Berichte, die von den Missionaren oder Chinareisenden erhältlich waren, und begann selbst eine umfassende Korrespondenz, die bis in sein Todesjahr 1716 anhielt. Diese war thematisch 
breit gefächert und umfasste naturwissenschaftlich-technische neben philosophischen Themen, die chinesische Schrift, Sprache, Politik und das Alltagsleben. Im Jahr 1689 hatte er die Schrift Novissima Sinica veröffentlicht. Zeitlebens brachte er China die grösste Hochachtung entgegen. Er zog das Fazit, «dass die höchste Kultur und die höchste technische Zivilisation der Menschheit heute gleichsam gesammelt sind an zwei äussersten Enden unseres Kontinents, in Europa und Tschina, das gleichsam wie ein Europa des Ostens das entgegengesetzte Ende der Welt ziert» (Novissima Sinica, zit. bei Buschmann, 2009). Besondere Achtung zollte Leibniz dem sozialen Verhalten der Chinesen, da sie alles verachteten, was bei den Menschen Aggressionen erzeuge und fördere. Er glaubte, dass Chinesen die Europäer in den Regeln des kultivierten Lebens überträfen und machte den berühmten Vorschlag, dass sie - als Gegengewicht zu den Jesuiten - nach Europa kommen sollten, um eine "praktische Philosophie und vernunftgemässe Lebensweise» (Buschmann, 2009) zu lehren. Leibniz' und auch der Jesuiten Verehrung der chinesischen Kultur traf in Europa auf grosses Interesse. Am Schluss soll dieser Strang noch einmal aufgegriffen werden.

Dieser Beitrag ist einem Kulturaustausch gewidmet, der in den 80erJahren des letzten Jahrhunderts begann, als sich China nach jahrzehntelanger Abschottung und den traumatisierenden Umwälzungen der Mao-Ära ökonomisch und kulturell wieder in Richtung Westen öffnete. Psychologie und Psychiatrie waren nach langer Isolation rückständig, psychische Störungen waren während der Kulturrevolution als Folgen «falscher Ideologie» oder «falschen Klassenbewusstseins» angesehen worden. Im Jahr 1988 begann ein einzigartiges deutsch-chinesisches Psychotherapieprojekt, das den Hintergrund für die folgenden Ausführungen bildet. Das Projekt, an dem bis heute verschiedene Psychotherapieschulen (Verhaltenstherapie, Familientherapie und psychoanalytisch orientierte Psychotherapie) beteiligt sind, wird ausführlich in Zhong De Ban oder: wie die Psychotherapie nach China kam (Simon et al., 2011) beschrieben. Im Jahr 1995 wurde in der Deutsch-Chinesischen Akademie für Psychotherapie (www.dcap.de) ein institutioneller Rahmen geschaffen (Haßß-Wiesegart \& Schweitzer, 2004). Erstmalig wurden in der Volksrepublik China systematische Ausbildungsgänge in Psychotherapie angeboten, die von den deutschen Kollegen erarbeitet worden waren. Ich nahm als Dozentin für psychoanalytisch orientierte Psychotherapie teil. Von 2000 bis 2008 konzentrierte ich meine Lehrtätigkeit auf ein grosses psychiatrisches Krankenhaus, das Shanghai Mental Health Center, das eine grosse psychotherapeutische Ambulanz und eine Psychotherapiestation unterhält. Unterstützt durch ein Stipendium des Deutschen Akademischen Austauschdiensts 
fuhr ich regelmässig jährlich nach Shanghai; mein längster Aufenthalt dauerte vier Monate. Ziel unserer Bemühungen war und ist es, den chinesischen Kollegen zu helfen, Kenntnisse zu erwerben, um dem grossen Bedarf an Psychotherapie in China entgegenzukommen. In vielen Familien zeigen sich Spuren schwerer, historisch bedingter Traumatisierungen (Haag \& Zhao, 2004). Darüber hinaus befindet sich die Volksrepublik China in einer massiven gesellschaftlichen und kulturellen Umwälzung, die mit einer neuen Kulturrevolution vergleichbar ist. Das Interesse der chinesischen Kollegen an unserem Ausbildungsangebot war und ist gross; insbesondere die Kurse in psychoanalytisch orientierter Psychotherapie sind bis heute sehr gefragt (Gerlach, 2010). Im Jahr 2004 wurde eine Psychoanalytische Vereinigung Chinas mit 33 Mitgliedern gegründet. Die Internationale Psychoanalytische Vereinigung unterstützt die Entwicklung der Psychoanalyse in China und hat dies 2010 mit der Ausrichtung eines in Beijing ausgetragenen Kongresses Freud in Asia bekräftigt. Auf dem Weltkongress 2011 wurde der erste chinesische Kollege begrüsst, der nach den Kriterien der Internationalen Psychoanalytischen Vereinigung ausgebildet worden ist.

Ich werde im Folgenden über Beobachtungen und Erfahrungen berichten, die ich in den Jahren von 1988 bis 2008 während meiner Lehrtätigkeit in China gemacht habe (Haag, 2011). Ich bin mir bewusst, dass ich in meinen Ausführungen als jemand, die im Westen sozialisiert worden ist, befangen bin und dass ich ausserdem vieles - zum Zweck der Klarheit - vereinfachen muss. Dennoch soll derVersuch gemacht werden, am Beispiel China zu verstehen, wie unterschiedliche Kulturen jeweils eigene seelische Strukturen bilden und wie sich westliche Therapieformen dazu verhalten.

Schwerpunktmässig geht es im Folgenden um die unterschiedlichen Konstruktionen des Selbst und seinen spezifischen kulturellen Ausformungen in Ost und West, die dann in einen Bezug zu grundlegenden psychoanalytischen Konzepten gesetzt werden. Weiter werden kulturimmanente Besonderheiten wie Schamanfälligkeit und Harmoniestreben aus psychoanalytischer Sicht beleuchtet. Schliesslich soll die Frage eines Transfers psychoanalytisch orientierter Psychotherapie in eine ostasiatische Kultur behandelt werden.

\section{Das Selbst als kulturelles Konstrukt}

Es gibt keinen Zweifel darüber, dass unterschiedliche Kulturen jeweils charakteristische Persönlichkeitsmuster hervorbringen (Bond \& Hwang, 1986; Triandis et al. ,1988; Markus \& Kitayama, 1991, 1998; Heine, 2001). Das Konstrukt «Selbst» umfasst körperliche, seelische und soziale Persönlichkeitsaspekte, die 
durch kulturelle und/oder historische Bedingungen geformt werden, die die Sozialisation eines Menschen ausmachen. Wir werden durch Gewohnheiten und Sitten, durch Mythen, Traditionen und Lebensstil bestimmt. Das Selbst umfasst die Eigenwahrnehmung in einem bestimmten sozialen Bezugsrahmen, in dem man sich selbst erlebt oder auch von anderen wahrgenommen wird (Tam, 1995). Es ist also zweifach determiniert; es ist sowohl Subjekt als auch Objekt (Johnson, 1985). Diese duale Natur des Selbst impliziert, dass man sich einerseits selbst zum Objekt seinerWahrnehmung machen, sich selbst andererseits aber auch gleichsam durch die Brille der Anderen betrachten kann. Auch wenn Markus und Kitayama (1991) in ihrem klassischen Aufsatz "Culture and the Self” betonen, dass bestimmte Aspekte des Selbst universal seien, ordnen sie doch gleichzeitig bestimmte Eigenschaften verschiedenen Kulturen zu. So unterscheiden sie das westliche "independent", also unabhängige, vom östlichen “interdependent” oder vernetzten Selbst.

In unserer westlichen Kultur ist Unabhängigkeit oder Autonomie ein hoch besetzterWert, der auch psychische Gesundheit mit definiert. In einer individuumzentrierten Welt wird die erfolgreiche Persönlichkeitsentwicklung daran gemessen, ob der Grundkonflikt zwischen Abhängigkeitswünschen und Autonomiestrebungen gelöst werden kann. Das Ideal ist eine abgegrenzte, konsistente, eigenständige Persönlichkeit, die sich in verschiedenen Situationen nicht ändert und unverwechselbar bleibt. Gegen den Strom zu schwimmen, löst eher Bewunderung als Kritik aus. Selbstbestimmtheit und Selbstverwirklichung sind hoch geschätzt. Es gibt eine scharfe Trennung zwischen den Vorstellungen, die man von sich selbst und von anderen hat. Der Individuationsprozess findet vor allem in der Adoleszenz statt, die in China anders gestaltet ist als im Westen. Hier beobachten wir eine relativ lange Abhängigkeit der Jugendlichen und jungen Erwachsenen von ihren Eltern und der gesamten Familie, die einen starken Einfluss auf ihre heranwachsenden Kinder ausübt. Bis heute hat der Begriff «individuell» eine mehr oder weniger negative Konnotation mit «selbstsüchtig» und «egoistisch» (Bond \& Hwang, 1986; Nisbett, 2003). In ostasiatischen Gesellschaften wird der Einzelne weniger als eigene Einheit wahrgenommen. Er ist Teil eines interagierenden sozialen Netzwerks - wie der Familie oder anderen Bezugsgruppen. Die Generation, die älter als 30 ist, ist in grossen Familien erzogen worden. Bis 1970 betrug die Fertilitätsrate mehr als 6 Kinder (Zeng, 1991). Grosseltern, Tanten, Onkel, Geschwister und Cousins haben engen Kontakt und ermöglichen multilaterale Identifikationsmöglichkeiten. Auch nach Einführung der Einkindpolitik werden die Kinder häufig von Eltern und Grosseltern gemeinsam aufgezogen, zumindest häufiger als im Westen. Wie die Steine einer Mauer unterstützen sich die Menschen gegenseitig und bilden eine 
stabile, verlässliche Gemeinschaft. Um den Zusammenhalt des Ganzen zu gewährleisten, muss der Einzelne seine eigenen Wünsche und Bestrebungen zugunsten der gemeinsamen Ziele unterdrücken, sonst besteht unter Umständen die Gefahr, dass die Mauer wackelt oder gar zusammenfällt. Entsprechend dem konfuzianischen Erbe hat die Gemeinschaft eine hierarchische Struktur. Das Selbstgefühl ist an einem Wir-Gefühl orientiert und dabei mit dem Ansehen, der Ehre und der Wertschätzung, die die Familie oder Bezugsgruppe erfährt, identifiziert. So beobachten wir in China selten Trennungen oder irreparable Zerwürfnisse unter den Familienmitgliedern. Die Selbst-Grenzen sind durchlässig.

Einige Beobachtungen sollen dieses illustrieren:

In die Ambulanz, in der ich arbeitete, kamen auch erwachsene Patienten kaum ohne Begleitung von Familienmitgliedern, Freunden oder Arbeitskollegen. Geheimnisse in den Familien sind kaum vorhanden. Mütter von Adoleszenten informierten die Therapeuten nicht selten über Gegebenheiten, die sie aus den Tagebüchern ihrer Kinder hatten. Im Kontrast zu westlichen Kleinkindern, die relativ früh separiert werden, schlafen chinesische Kinder oft - unabhängig von der Grösse der Wohnung - im gleichen Raum wie die Eltern. Offenbar ist es nicht ungewöhnlich, dass auch Kinder in der Pubertät oder sogar Adoleszenz das Schlafzimmer, wenn nicht gar das Bett, mit einem Elternteil, meistens der Mutter, teilen.

Studien zeigen, dass chinesische Eltern ihre Kinder hinsichtlich Selbstständigkeit und Verantwortlichkeit wenig unterstützen (Ching, 1997). Wenn man dabei berücksichtigt, dass das Ansehen der Familie mit einer erfolgreichen Entwicklung der Kinder verknüpft ist, so finden wir zwischen Eltern und Kindern eine gegenseitige Abhängigkeit - oder, wie Markus und Kitayma es nennen, eine Interdependenz. Diese bestimmt ebenso die Selbstbildung wie den kulturellen Wert gruppenorientierten Verhaltens. Anschaulich beschreibt der Japaner Mori Jori die unterschiedliche Konstellation des östlichen und des westlichen Selbst. Er vergleicht das westliche mit einer harten Eierschale, die ihm Kohärenz verleiht, das östliche dagegen hat für ihn eine weiche, flexible Membran (zit. nach Creighton, 1990, S. 294). Das asiatische Selbst kann sich schnell an verschiedene Umstände anpassen und entsprechend des jeweiligen Kontextes umstellen.

Diese unterschiedlichen Konstrukte haben eine grosse Bedeutung für das Alltagsleben - und auch das psychotherapeutischeVerständnis. Als Beispiele wende ich mich jetzt dem Kommunikationsstil und dem «Gesichtsverlust» zu. 


\section{Kommunikation}

Der amerikanische Anthropologe Edward T. Hall (1989) unterscheidet Kulturen nach ihrem Kommunikationsstil. Er beschreibt zwei Idealtypen, nämlich Kommunikation mit hohem und mit niedrigem Kontext. Zwischen diesen Typen gibt es fliessende Übergänge. In China und anderen asiatischen Ländern beschreibt er einen Kommunikationsstil mit hohem Kontext als kulturspezifisch, während er diesen in den USA und den meisten europäischen Ländern als einen mit niedrigem Kontext sieht. Vor dem Hintergrund der unterschiedlichen Selbst-Konstrukte ist dies unmittelbar einleuchtend: Die fliessenden Grenzen zwischen Menschen in einer eng miteinander verbundenen, kollektivistischen Gesellschaft erlauben eine «implizite» Verständigung. Die Menschen teilen die gleichen Informationen, sie müssen oft nicht viel erklären und verstehen sich oft intuitiv auf einer gleichsam «symbiotischen» Ebene. Sie haben auch eine sehr viel grössere Toleranz für Widersprüche, akzeptieren Unvereinbarkeiten und denken dialektisch (English \& Chen, 2007). Die Einheit der Gegensätze, wie sie im Kreis, der Yin und Yang umschliesst, symbolisiert ist, ermöglicht eine hohe Ambiguitätstoleranz. Hier einige Beobachtungen aus meiner Lehrtätigkeit:

Manchmal, wenn es für meine Schüler schwierig war, ihre Eindrücke über Patienten zu beschreiben, bot ich Alternativen an, die sich für mein Gefühl ausschlossen, zum Beispiel, ob sie sie sich eher erfreut oder verärgert über bestimmte Verhaltensweisen fühlten. Als Antwort bekam ich ein «Ja» oder auch ein «Nein». Offensichtlich konnten sie ihre verschiedenen Emotionen problemlos integrieren, was mir sehr viel schwerer fiel. Ich hatte auch immer wieder Schwierigkeiten, in den Fallvorstellungen einen roten Faden zu finden, der mir bei der Entwicklung eines psychodynamischen Verständnisses hätte helfen können. Ich war es nicht gewohnt, implizite Botschaften gut zu verstehen. Manchmal hatte ich den Eindruck, dass die Kollegen nicht auf den Punkt kamen, und hatte das dringende Bedürfnis, zu strukturieren.

Als jemand, die in einer Kultur mit einem Kommunikationsstil mit niedrigem Kontext aufgewachsen ist, bin ich es gewohnt, linear zu denken und mich explizit zu äussern. Unschärfe und Ambiguitäten werden vermieden. Wir bemühen uns, Widersprüche zu lösen, und sind hinsichtlich Vagheiten und Unsicherheiten nicht besonders tolerant.

\section{Angst vor Gesichtsverlust und Scham}

Scham und die Angst, das Gesicht zu verlieren, gehören zusammen und sind kontingent. In einer Kultur, in der Menschen eng miteinander verbunden sind, ist 
Harmonie nur möglich, wenn der Einzelne sich allgemeinen Regeln anpasst und im sozialen Kontext bestimmte Rollen übernimmt. Nonkonformismus gefährdet die Harmonie. Menschen, die sich nicht anpassen können oder gar exponieren, werden ausgeschlossen und schämen sich. Sie verlieren ihr Gesicht - und nicht nur sie, sondern auch ihre Bezugsgruppe. Die Schamangst ist in allen Gesellschaften ein wichtiges Korrektiv in der sozialen Ordnung, in kollektivistischen Gesellschaften ist dieses jedoch besonders ausgeprägt. Die Angst, die der Emotion Scham immanent ist, ist die des Ausgestossen- oder Verlassenwerdens.

Leistung und Ausbildung spielen in chinesischen Familien eine grosse Rolle, insbesondere heutzutage in der zunehmend konkurrenzorientierten Gesellschaft. Für chinesische Studenten ist es schwer, offen zuzugeben, wenn sie dem Unterricht nicht folgen können. Sie befürchten so einen Gesichtsverlust - oder sie wollen auch den Gesichtsverlust des Lehrers vermeiden, der das Wissen offensichtlich nicht gut vermitteln konnte.

Ich habe einmal in einer Diskussion bemerkt, dass ich Probleme hätte, einen vorgestellten Fall psychoanalytisch ausreichend zu verstehen. Nach der Stunde kam eine Kollegin zu mir, die mir sagte, dass sie mich bewundere. Für einen chinesischen Lehrer, so meinte sie, wäre ein solches Bekenntnis sehr schwer gewesen. Vielleicht hatte ich mit dieser Bemerkung für meine Schüler einen Gesichtsverlust erlitten, weil ich mich nicht als Expertin gezeigt hatte.

Als die Anthropologin Ruth Benedict 1946 die japanische «Schamkultur» der US-amerikanischen «Schuldkultur» gegenüberstellte, wurde das von japanischen Sozialwissenschaftlern protestierend zur Kenntnis genommen (Creighton, 1990). Der Grund dafür war, dass Schuld als soziales Verhaltensregulativ auf einer höher entwickelten psychischen Ebene angesiedelt wurde, nämlich als Konsequenz verinnerlichter moralischer Standards. Scham geht der Entwicklung des ÜberIch voraus. Sie entsteht aus der Unfähigkeit, Idealen oder Erwartungen nicht zu entsprechen, und die damit verbundene Angst ist die vor Entwertung oder Demütigung und schliesslich Ausschluss. Die beschämte Person glaubt sich beobachtet und blossgestellt. Ich halte diese Gegenüberstellung, besonders, wenn sie wertend vorgenommen wird, für problematisch. In jeder Kultur regulieren Scham und Schuld das Zusammenleben, wenn sicher auch andere Schwerpunkte gesetzt werden. Inzwischen haben auch asiatische Autoren beschrieben, dass Scham als Regulans in kollektivistischen, Schuld in individualistischen Gesellschaften vorherrsche (Creighton, 1990). In Anbetracht der unterschiedlichen Struktur des Selbst ist dies auch leicht nachvollziehbar. Das stärker aussengeleitete, am Umfeld orientierte, interdependente Selbst mit starkem Druck, sich an die Normen der 
jeweiligen Gruppe anzupassen, hat immanent eine stärkere Neigung, schamvoll zu reagieren. Das Selbst in individualisierten Kulturen orientiert sich ebenfalls an sozialen Regeln, die die internalisierten Standards der primären Bezugspersonen repräsentieren und den Kern des Über-Ich bilden. Diese Internalisierung jedoch führt zu einer stärkeren Unabhängigkeit, die Verantwortung liegt beim Einzelnen, der nur sich selbst Rechenschaft schuldig ist - sofern er andere nicht schädigt. Die primäre Angst bezieht sich nicht so sehr auf das Verlassenwerden als auf Bestrafung durch fantasierte Elternfiguren oder ein schlechtes Gewissen.

\section{Psychoanalytisch orientierte Psychotherapie in China}

Im Folgenden sollen beispielhaft einige psychoanalytische Grundannahmen behandelt werden, die mit dem ostasiatischen Selbst-Konstrukt in Widerspruch geraten können.

\section{Der therapeutische Rahmen, Neutralität und Abstinenz}

Die Grundpfeiler der Theorie psychoanalytischer Behandlungstechnik sind: ein verlässliches Setting mit einem stabilen Rahmen und eine neutrale Haltung dem Patienten gegenüber, die einschliesst, dass der Therapeut sich versagt, die Wünsche und Bedürfnisse seines Patienten unmittelbar zu erfüllen. Für alle, insbesondere aber weniger erfahrene Therapeuten, kann dieses zu einer Herausforderung werden. Die Rolle des analytisch orientierten Therapeuten ist äusserst widersprüchlich: Zum einen muss er eine exklusive, intime, vertrauensvolle Situation schaffen - zum anderen muss er Abstand halten. Mehr noch: Dieser Abstand, der durch die neutrale Haltung erst möglich wird, wird zurVorbedingung für diese intime Situation. Die Neutralität, in der weder Partei ergriffen wird, noch be- oder verurteilt wird, macht es erst möglich, dass der Patient sich offen und angstfrei äussern kann. Voraussetzung ist natürlich, dass der Therapeut eine gute, unterstützende Atmosphäre bereitstellt, in der der Patient das Gefühl bekommt, dass er verstanden wird, ohne bewertet zu werden.

In China, wo die Selbst-Grenzen durchlässiger und die Menschen stärker miteinander vernetzt sind, können diese Aspekte zu einem Problem werden. So kann zum Beispiel ein chinesischer Therapeut in Konflikt geraten, wenn er gebeten wird, jemanden in Behandlung zu nehmen, der zu seinem inneren oder äusseren Kreis gehört. Die Zurückweisung eines solchen Patienten kann zu einem Affront werden, der nicht mit dem Anspruch harmonischer Beziehungen in Einklang zu bringen ist. Die Verschränkung privater und beruflicher Beziehungen kann zu einem therapeutischen Dilemma werden. Auch die chinesische Sitte, Dankbarkeit 
in Form von Einladungen oder Geschenken, insbesondere Geldgeschenken, auszudrücken, kann zu einem Problem werden. Diese Dankbarkeitsgesten zurückzuweisen, kann als Unhöflichkeit gesehen werden - und einen Gesichtsverlust für diejenigen bedeuten, die sich verpflichtet fühlen.

Aus chinesischer Sicht kann die Exklusivität der therapeutischen Beziehung als bedrohlich oder angsterregend für die Familie des Patienten erlebt werden. Es ist nicht ungewöhnlich, dass Verwandte - oder sogar Vorgesetzte - sich in die Therapie einzumischen versuchen, zum Beispiel, wenn sie dem Therapeuten gewisse Informationen über den Patienten geben möchten - oder sich auch beklagen, wenn sie eine Besserung der Symptomatik vermissen. Vor diesem Hintergrund wäre es möglicherweise sinnvoll, vor Therapiebeginn eine Sitzung mit den wichtigen Vertrauenspersonen des Patienten anzuberaumen, um über die basalen Voraussetzungen einer psychoanalytisch orientierten Psychotherapie zu informieren. So können Missverständnisse vermieden und Vertrauen gewonnen werden.

\section{Introspektion, Empathie und Übertragung}

Kakar (1985) weist darauf hin, dass das sokratische «Erkenne dich selbst» als geistige Matrix westlicher, einsichtsorientierter Psychotherapien gelten kann. Dies setzt die Vorstellung voraus, dass der Mensch eine einzigartige, untrennbare Einheit sei. Introspektion oder "psychological mindedness" ist aus dem philosophischen Erbe des Westens entstanden. In China hat dies keine Tradition, was chinesische Psychiater (Lin, 1983, zit. nach Ng, 1985; Tung, 1991; Tseng, 2005) veranlasst hat, zu betonen, dass einsichtsorientierte Psychotherapien für China weniger geeignet seien. In einer 2007 veröffentlichten Studie mit 342 Studenten der Universität Lanzhou fand sich bei diesen im Vergleich zu einer US-amerikanischen Stichprobe ein signifikant geringerer Wert in der "Psychological Minded Scale" (Hua et al., 2007). Nach meinen Erfahrungen kann dieser Befund nicht verallgemeinert werden, aber möglicherweise kann er doch aus der unterschiedlichen Konstruktion des Selbst abgeleitet werden.

Introspektion, die Fähigkeit, sich gleichzeitig selbst $\mathrm{zu}$ erleben $u n d \mathrm{zu}$ beobachten, ist nur durch eine Ich-Spaltung denkbar. Diese ermöglicht, dass die Person sowohl Subjekt als auch Objekt ist und einen inneren Dialog mit sich selbst beginnen kann. In der therapeutischen Situation ist dies die Voraussetzung für die Motivation, die dysfunktionalen Aspekte der Persönlichkeit zu verändern. Diese innere Motivation des Patienten ist in der Therapieforschung der wichtigste Prädiktor für den Therapieerfolg. Fehlt die Introspektionsfähigkeit, so muss es die primäre Aufgabe des Therapeuten sein, ihm zu helfen, eine solche zu ent- 
wickeln und ihn dorthin zu führen. Falls das nicht gelingt, kann eine Therapie nach psychoanalytischen Kriterien nicht erfolgen, und der Therapeut muss sehen, ob der Patient nicht möglicherweise von einem mehr unterstützenden Vorgehen mehr profitieren könnte als von einem einsichtsorientierten. Empathie bedeutet Kommunikation sowohl mit den bewussten als auch unbewussten Gefühlen des Anderen. Empathie und Mitleid sind einander nah und werden oft verwechselt. In der Therapie können wir uns nur durch Empathie - nicht Mitleid - die unbewussten Gefühle des Patienten erschliessen. Die Voraussetzung für empathisches Verstehen ist eine vorübergehende Identifikation mit dem Patienten - ohne sich $\mathrm{zu}$ verlieren oder seine eigenen Gefühle in ihn zu projizieren. «Um empathisch zu sein, muss das Individuum in der Lage sein, sich selbst von seinen Gefühlen zu distanzieren - sodass es ihre Ursprünge und die Bedeutung im jeweiligen Zusammenhang erkennen kann» (Basch, 1983, S. 119). Dieses ist nicht einfach, weil wir oft unsere eigenen Gefühle im Patienten finden, Aspekte unseres eigenen Lebens, unserer Hoffnungen, Träume, unseres Leidens - und es ist dann essenziell, Subjekt von Objekt zu differenzieren. Das ist besonders wichtig, wenn wir mit eigenen Eigenschaften konfrontiert sind, die wir nicht mögen oder die wir bekämpfen müssen. Die Fusion zwischen den Gefühlen von Therapeut und Patient kann ein konstruktives Arbeitsbündnis stören. Die Versuchung, sich ganz mit dem Patienten zu identifizieren, ist gross - oder, im Gegenteil, ihn abzulehnen. Wegen der gemeinhin fliessenden Grenzen zwischen Subjekt und Objekt kann dies in China zu einem kulturspezifischen Problem werden, wie ich es in meinen Fallbesprechungen nicht selten erlebt habe. So war mir manchmal nicht klar, ob die Kollegen von sich oder ihren Patienten berichteten.

Auch zum Erkennen und Analysieren von Übertragung und Gegenübertragung ist eine Ich-Spaltung nötig. Der Therapeut muss zwischen den aus kindlichen Beziehungsmustern herrührenden Projektionen und den «realen» Personen unterscheiden können und dies für sein Verständnis der psychodynamischen Konstellation nutzen. Dies ist manchmal schwierig, besonders, wenn der kulturelle Hintergrund durch eine konfuzianische Tradition geprägt ist, in der Hierarchie und Harmonie konstitutive Elemente sind. Konflikte oder eine negative Übertragung zum Ausdruck zu bringen, kann für Patienten zum Problem werden. Sie möchten ihrem Therapeuten Respekt zeigen, um Sympathie und Harmonie aufrechtzuerhalten und um der traditionell tief verwurzelten Pflicht zur Kindesloyalität genüge zu tun. Sie neigen dazu, ihn zu idealisieren, und er muss diesen kulturell gebahnten Widerstand aufdecken. In ihren Fantasien statten die Patienten ihren Therapeuten mit viel Macht aus. Er repräsentiert den sorgenden Vater oder die 
Mutter, die für das Wohlergehen verantwortlich sind. Dieses finden wir selbstverständlich auch in westlichen Behandlungen, aber der Umgang mit diesen Wünschen kann unterschiedlich sein. Der westliche Therapeut wird vorsichtig ablehnen, kindliche Wünsche zu erfüllen und den Patienten ermutigen, eigene Ressourcen zu mobilisieren, um seine Autonomie zu entwickeln. Für chinesische Therapeuten ist es schwerer, den Patienten zu frustrieren und zu enttäuschen - und dieses geht oft nicht ohne Gefühle von Schuld und Hilflosigkeit ab. Besonders in diesem Punkt wird die Problematik des Transfers analytisch orientierter Therapie evident: Eigenständigkeit und Handlungsfreiheit, die klassischen Ziele psychodynamischer Therapien, können in gemeinschaftsbezogenen, interdependenten Kulturen infrage gestellt werden. Dieses mag sich im Zuge der die wirtschaftliche nachziehende kulturellen Globalisierung verändern - aber das wird sicher noch einige Generationen dauern.

Für alle hier beschriebenen Aspekte ist die Notwendigkeit einer IchSpaltung der gemeinsame Nenner. Nach meiner Erfahrung haben chinesische Therapeuten Probleme damit. Ich möchte hier wieder nicht generalisieren, aber es gibt im Vergleich zu meiner Supervisionsarbeit im Westen Unterschiede. Oft waren chinesische Patienten für eine psychodynamische Therapie nicht ausreichend indiziert, auch von erfahreneren Therapeuten. Viele Therapien blieben ohne Erfolg - oder die Patienten kamen nicht wieder. Häufig fehlte es an Introspektion oder psychologischem Interesse, was die Kollegen nicht ausreichend untersucht hatten. Sie arbeiteten dann rein supportiv - und fühlten sich nach der Supervision verunsichert. Ich war es ebenfalls, bis ich begriff, dass es sich offensichtlich um ein zugrunde liegendes kulturelles Phänomen handelte, das mit der unterschiedlichen Bildung des Selbst verbunden war.

Um die Differenzen zusammenzufassen:

Für das westliche, stärker abgegrenzte Selbst ist es charakteristisch, dass es sich in unterschiedlichen Situationen kaum verändert. Dagegen steht das asiatische, flexible Selbst, das sich leicht verschiedenen Situationen anpassen kann, wahrscheinlich durch seine Tendenz, sich zu identifizieren - oder zu fusionieren. Identifikationen werden jedoch nicht wahrgenommen, weil die Grenzen fliessend sind und ein notwendiger Abstand zwischen Beobachter und Beobachtetem schwer hergestellt werden kann. Objekt und Subjekt, bzw. ihre Repräsentanten, sind also nicht ausreichend separiert, um ein psychisches Oszillieren zwischen ihnen zu erlauben. Bei der Introspektion wird ein Teil des Selbst zum beobachteten Objekt - und die Teile kommunizieren miteinander. Empathie verbindet die temporäre Identifikation mit einem komplexen reflektierenden oder kognitiven 
Prozess, um das innerseelische Erleben des Patienten zu verstehen, ohne selbst zu stark involviert zu werden. Auch dies ist nur über eine Spaltung möglich, die darüber hinaus ebenfalls nötig ist, um Übertragungs- und Gegenübertragungsaspekte von der Realperson des Therapeuten oder des Patienten zu differenzieren.

\section{Eine kulturspezifische Abwehr: die passive Rationalisierung - oder die Ah-Q-Mentalität}

Wenn die intrapsychischen Mechanismen der Angstbewältigung auch universell sind, so variiert die Art der Ängste doch. Es ist nicht verwunderlich, dass ein relationales, konformistisches Selbst andere Anpassungs- oder Abwehrmechanismen entwickelt als ein stärker abgegrenztes Selbst.

Chinesische Autoren (Tseng 2005; Xiao 2008; Cheung et al., 2005) haben einen spezifischen chinesischen Konfliktlösungsmodus hervorgehoben, die passive Rationalisierung oder die Ah-Q-Mentalität. «Ah-Q» wurde 1921 von dem grossen Schriftsteller Lu Xun erfunden, um auf satirische Weise den chinesischen Nationalcharakter zu beschreiben. In seiner Geschichte ist Ah-Q ein armer Tropf, der zahlreiche Niederlagen und Demütigungen erfährt, ohne darauf zu reagieren oder wütend zu werden. Er unterdrückt nicht nur seinen Ärger, sondern ist sogar in der Lage, Niederlagen als Siege zu verkaufen. Noch 90 Jahre nach seiner Erfindung wird das Verhalten von Ah-Q (auf Deutsch nachzulesen in Lu 2006 unter dem Titel: Die wahre Geschichte des Herrn Jedermann) als «typisch chinesisch» angesehen. Um kulturspezifischeWesenszüge zu erfassen, wurde im chinesischen Persönlichkeitsinventar, dem Chinese Personality Assessment Inventory, das zuletzt 2001 standardisiert wurde, die Ah-Q-Mentalitätsskala eingeführt, um einen psychischen Mechanismus zu beschreiben, der Problemlösung durch Passivität beschreibt.

Während meiner Tätigkeit als Lehrtherapeutin erlebte ich gelegentlich von mir schwer nachzuvollziehende Situationen. Auch wenn einige - besonders männliche - Kollegen ihre Passivität selbst als Hauptproblem vortrugen, war es für sie schwer - wenn nicht unmöglich - das zu ändern. So war ich überrascht, dass junge Kollegen, denen ich Selbsterfahrungssitzungen anbot und die wenig über ihre Kindheit wussten, nicht ihre Eltern, mit denen sie in ständigem Kontakt waren, fragten - zum Beispiel wo sie früher lebten oder wer sich um sie während der turbulenten Jahre ihrer Kindheit, die meist in die Zeit der Kulturrevolution fiel, gekümmert hatte. Auch die aus den Sitzungen gewonnenen Einsichten wurden selten handelnd ausprobiert. 
Es wäre wohl ein ethnozentrischer Fehler, dieses Verhalten nur als individuellen Widerstand zu verstehen. Vielmehr scheint dieser Mangel an Neugier und einem Interesse an aktiver Klärung tief in der chinesischen Kultur verankert. Die scheinbar geringe Motivation, sich zu verändern, war für mich, die ich an aktivere Verhaltensmuster gewöhnt bin, eine Herausforderung.

Der französische Philosoph F. Jullien (2006), der die chinesische Kultur gut kennt, beschreibt den fundamentalen Unterschied zwischen westlichem und chinesischem Denken. Das westliche sei linear, zielorientiert, vorsätzlich. Das Subjekt ist der Hauptakteur bei der Veränderung einer Situation - oder bei dem Versuch, eine Wirkung zu erreichen. Der das Handeln in China bestimmende, daoistische Weg sei mühelos, ohne Anstrengung oder grössere Aktivität - man verlasse sich auf das Situationspotenzial bzw. die Möglichkeiten, die gegebenen Konstellationen immanent sind. Wu Wei, Handeln durch Nicht-Handeln, heisst die daoistische Devise. Für uns westliche Menschen ist es fremd, sich auf die selbstregulierenden Lebensprozesse zu verlassen; wir müssen immer eingreifen. Wir müssen uns aber auch fragen, ob das gleichmütige Vertrauen der Chinesen nicht weiser ist.

Um noch einmal auf meine Eindrücke als Dozentin zurückzukommen: Ich traf oft auf eine von mir als regressiv wahrgenommene Erwartungshaltung, nicht nur in den Selbsterfahrungssitzungen, sondern auch in den vorgestellten Patientengeschichten. Vor dem Hintergrund des gerade Beschriebenen ist dieses Muster nun gut nachzuvollziehen. Beitragen zu dieser Haltung wird auch das konfuzianische Erbe einer hierarchischen Kluft zwischen Therapeut und Patient oder Lehrer und Schüler. Beide, Patienten und Schüler, erwarten, dass sie Ratschläge und Erklärungen von den Älteren bekommen - und es ist nicht ganz einfach für chinesische Therapeuten, sich ihrer traditionellen Rolle zu entziehen und die Eigenständigkeit des Patienten zu stimulieren und zu fördern. Statt zu beraten oder zu erklären wird der westliche Therapeut versuchen, die Gefühle bewusst zu machen, versuchen, zu verstehen und zu deuten und so den Reifungsprozess, der als Ziel die Unabhängigkeit vom Therapeuten hat, begleiten. Deshalb wird er regressive Wünsche möglichst nicht erfüllen.

Darüber werden auch westliche Patienten manchmal enttäuscht und ärgerlich, aber chinesische Patienten werden mit einer Verweigerung konfrontiert, die ihren kulturell gefärbten Erwartungen diametral entgegengesetzt sind.

In seinem Buch über die Einführung systemischer Familientherapie in China beschreibt Zhao (2002) die Haltung chinesischer Therapeuten als moralisch, kontrollierend, aktiv und manipulierend. Die westliche fand er neutral, distan- 
ziert, neugierig und rational. Beide Haltungen sind Ausdruck unterschiedlicher kultureller Prämissen.

\section{Noch einmal: Gesichtsverlust und Scham}

Auf der ganzen Welt hat das traditionelle Image eines Arztes oder Therapeuten Züge von Autorität - und oft ist dies ein Vorzug. Dennoch: In Psychotherapien kann dies zu einer andauernden Abhängigkeit von einem idealisierten Übervater kommen, besonders in einer Kultur, in der eine hierarchische Struktur eine lange Tradition hat. Bei unterschiedlichen Meinungen kann es dem Patienten schwerfallen, zu widersprechen - oder er ist pseudokooperativ - nicht zuletzt, um den Therapeuten vor einem Gesichtsverlust zu schützen (Zhao, 2009). Ich erinnere mich, dass ich mich gelegentlich unbehaglich fühlte, wenn ich in die zweifelnden oder auch irritierten Gesichter meiner Schüler blickte oder andere nonverbale Zeichen etwa während einer Selbsterfahrungssitzung wahrnahm. Wenn ich dieses spiegelte oder mit meiner Verunsicherung in der Gegenübertragung konfrontierte, konnte das meistens geklärt werden, aber sicher nicht immer. Andererseits können auch Patienten die Befürchtung haben, ihr Gesicht zu verlieren, wenn sie Annahmen, Konfrontationen oder Deutungen nicht verstehen oder akzeptieren können. Hier kann dann insofern ein Widerstand zum Zuge kommen, als sie Einverständnis vorgeben, Kooperation suggerieren - oder die Behandlung einfach abbrechen. Diese Erfahrung habe ich bei den jungen chinesischen Kollegen und ihren Patienten häufiger gemacht und war dann erstaunt, wie wenig sie dies zu tangieren schien. Möglicherweise war dies ein stillschweigenderVertrag der beiden Seiten, um das Gesicht zu wahren.

\section{Schlussbetrachtungen}

Zweifellos entwickeln sich in verschiedenen Kulturen unterschiedliche Ausgestaltungen des Selbst. In einer konfuzianistisch geprägten, gruppenbezogenen Tradition treffen wir auf ein Selbst mit fliessenden Grenzen, die dem Einzelnen ermöglichen, situationsabhängig in verschiedene Rollen zu schlüpfen. Tam (1995) spricht von einem «Rollenselbst», das in Familie und Gesellschaft pflichtgetreu seine erwarteten unterschiedlichen Aufgaben übernimmt. Wie ich versucht habe aufzuzeigen, gibt es verschiedene Implikationen, wenn wir die Frage der Kompatibilität dieses Selbst mit psychoanalytischen Behandlungsmethoden stellen. Wahrscheinlich liegt der wichtigste Unterschied zum westlichen, individualisierten Selbst in einer inhärenten, weniger ausgeprägten Fähigkeit, eine therapeutische Ich-Spaltung herzustellen, die die Voraussetzung für Empathie, Introspektion 
und das Erkennen von Übertragungs- und Gegenübertragungsphänomenen ist. Auch die Exklusivität der Therapeut-Patient-Beziehung ebenso wie die Faktoren Neutralität und Abstinenz sind eine Herausforderung bei dem Transfer von Psychoanalyse in eine sehr unterschiedliche Kultur.

In unseren häufig geführten Diskussionen, ob die Einführung von psychoanalytischer Psychotherapie nach China überhaupt angemessen sei, gab es verschiedene Argumente, diesen kulturellen Balance-Akt zu rechtfertigen. Ohne Zweifel befindet sich die chinesische Gesellschaft in einem Individualisierungsprozess. Dies ist die Folge gewaltiger Veränderungen, wenn nicht Revolutionen, wie dem Übergang von Plan- zu Marktwirtschaft, von Grossfamilien zu Einkindfamilien und dem langsamen Übergang von einer Agrar- in eine Industriegesellschaft. Im Zuge der Globalisierung beobachten wir einerseits eine Enttraditionalisierung (Giddens, 2001), auf der anderen Seite gibt es auch ein Wiederaufleben alter Wertvorstellungen, die während der Kulturrevolution zerstört worden waren.Gegen Ende des letzten Jahrhunderts begann die chinesische Regierung, sich wieder vermehrt dem Konfuzianismus zuzuwenden und Führer der Kommunistischen Partei Chinas erklärten ihn zur Hauptströmung in der chinesischen Kultur (Huntington, 1998). Als Präsident Hu Jintao 2005 die Partei aufrief, eine «harmonische Gesellschaft» zu etablieren, bezog er sich auf alte konfuzianische Werte (Bell, 2009). Aus westlicher Sicht sind wir mit Unvereinbarkeiten konfrontiert: Für uns schliessen sich Marktwirtschaft, Kommunismus und Konfuzianismus gegenseitig aus. Chinesischer Pragmatismus kann jedoch diese Widersprüche integrieren, sofern dies einem übergeordneten Ziel dient.

Auch wenn es viele Berichte über die «Verwestlichung» des Verhaltens, besonders urbaner junger Chinesen gibt, zeigen Studien doch, dass kulturelle Werte sich langsamer wandeln als das praktizierte Verhalten. Kindestreue und familiäre Verpflichtungen werden - unabhängig von der Qualität der innerfamiliären Beziehungen - immer noch hoch bewertet (Fuligni \& Zhang, 2004). Der Ödipus-Mythos der westlichen Welt wäre in einer konfuzianischen Gesellschaft nicht vorstellbar. In der chinesischen Parallelgeschichte tötet der Vater - wie in der griechischen Mythologie unwissentlich - seinen Sohn, als er nach 18 Jahren Abwesenheit von zu Hause zurückkehrt. Seine Macht ist unangetastet (Blowers, 2006).

Die Frage einer Übertragung psychoanalytischer Behandlung nach China ist nicht leicht zu beantworten. Ostasiatische Therapeuten betonen die Notwendigkeit technischer Modifikationen, um die Patienten zu erreichen (Tseng, 1995, 2005; $\mathrm{Ng}$, 1985). Sie schlagen statt des nichtdirektiven ein mehr erzieherisches, unter- 
stützendes Vorgehen vor. Zhong Youbin, ein führender Psychiater aus Peking, der 2009 verstarb, fühlte sich zur Psychoanalyse hingezogen. Er machte, wie Freud, eine «Selbst-Analyse» und liess einige elementare Ideen in seine therapeutische Technik einfliessen. So führte er aktuelle Probleme seiner Patienten auf ihre frühen Erfahrungen zurück, wobei er auch Familienangehörige als Erinnerungshelfer einbezog. Der Kern seiner Therapie lag darin, dem Patienten zu erklären, dass psychische Schwierigkeiten ihre Wurzeln in der Kindheit haben - und somit für Erwachsene nicht mehr gelten müssen. Sein Zugang geht über eine strukturierte und gelenkte Einsicht. Seine Behandlungsform wurde als «chinesische Analyse» oder auch «kognitive, einsichtsorientierte Psychotherapie» bezeichnet (Tung, 1991).

Ein weiterer Punkt mag für die kulturspezifische Persönlichkeitsentwicklung von Bedeutung sein. Im Vergleich zur westlichen ist die frühkindliche Sozialisation anders akzentuiert: Das erste Lebensjahr ist durch eine intensive orale Verwöhnung und eine grosse Nähe zur Mutter bestimmt. Es ist undenkbar, dass chinesische Babys in einem anderen Raum schlafen als die Mutter. In der analen Phase fehlt das bei uns betonte Schliessmuskeltraining; auch werden die Kinder weniger zur Selbstständigkeit ermuntert als bei uns. Aggressive Emotionen werden weniger toleriert; die Erziehung zu absoluter Loyalität wird eingeleitet. In der Latenzphase werden die Kinder streng angehalten, zu lernen; sie werden daran gewöhnt, Leistungen zu zeigen. Wie schon anfangs erwähnt, spielt auch in der Adoleszenz die Unterordnung unter die Eltern eine zentrale Rolle, und sie bleiben lange von diesen abhängig (Zhao, 2002).

Entscheidend ist das Wissen über kulturspezifische Erziehungspraktiken und ihre Bedeutung für die psychische Entwicklung - ebenso wie über den philosophischen Mutterboden der jeweiligen Kultur. Es ist sehr schwer, wenn nicht gar unmöglich, unseren ethnozentrischen Blick auszuschalten, wenn wir auf eine fremde Kultur blicken. Kultur-syntone Verhaltensvariationen sind kondensiert in Ich-syntonen Persönlichkeitszügen. Das Konzept der «reifen Persönlichkeit» ist von kulturimmanenten Gegebenheiten abhängig. Und dieses ist wieder mit einer eigenen Konstruktion des Selbst verbunden, das bestimmte charakteristische Verhaltensmuster formt.

Ob sich im Zuge der Globalisierung mit der zunehmenden Durchlässigkeit der Grenzen zwischen Ländern und Kontinenten tief verwurzelte kulturelle Eigenheiten verändern werden, wird die Zukunft zeigen. Für die Psychoanalyse stellt sich die Frage, ob sie «sinisierbar» ist, ohne ihre Grundannahmen zu opfern. Dieses zu untersuchen, wird eine wichtige Aufgabe sein, bei der auch vergleichende Kulturwissenschaftler einbezogen werden sollten. Blickt man derzeit auf die chi- 
nesische Psychotherapielandschaft, so finden wir einen grossen therapeutischen Pluralismus, nach dem alten maoistischen Motto: Lasst hundert Blumen blühen, lasst hundert Denkschulen miteinander wetteifern. Die von mir unterrichteten Kollegen machten meist parallel zu meinem Unterricht Ausbildungen in anderen Therapiemethoden. Ich kann das begrüssen, weil so die Möglichkeit eines für die Patienten massgeschneiderten Zugangs grösser wird. Es gibt auch chinesische Kollegen, die psychoanalytische Konzepte mit daoistischem oder buddhistischem Denken amalgamieren (zum Beispiel Zhang, 2007). Die Sicht auf andere Kulturen öffnet den Blick aber auch für unsere eigene. Unzweifelhaft ist die Berührung von Psychoanalyse und ostasiatischer Kultur eine grosse Herausforderung.

Wenn ich jetzt aus der Distanz auf meine Zeit als psychoanalytische Dozentin in China zurückblicke, bedauere ich sehr, dass meine kulturelle Kompetenz so begrenzt war. Psychotherapie ist generell in China mit seinem holistischen Menschenbild, historisch gesehen, eine ziemlich neue Errungenschaft, und ich gehöre in diesem Feld zur ersten Generation, die vermitteln durfte - und dies war eine faszinierende Erfahrung. Es tut mir jetzt leid, dass ich - aber auch meine Schüler - viel zu wenig Fragen gestellt haben. Das Autonomie-Ideal als Konsequenz unseres westlichen Denkens, das unsere Zielvorstellungen in der Psychotherapie bestimmt, kann in China bisher noch schwer gelten. Wir müssen uns aber auch die Frage stellen, ob die westliche Idealisierung von Eigenständigkeit und Selbstverwirklichung überhaupt noch zu unserer sozialen und psychischen Realität passt, und prüfen, ob damit nicht auch eine Entsolidarisierung verbunden ist, die der Gesellschaft schadet. Sich in Abhängigkeiten zu begeben, ohne dabei das Selbstwertgefühl zu verlieren, ist in unserer westlichen Kultur nicht leicht. Das Dilemma ist wahrscheinlich, dass beideWeltsichten, die östliche und die westliche, einseitig sind. Die westliche Überbetonung von Individualismus vernachlässigt die Gemeinschaft und Solidarität. Der östliche Kollektivismus vernachlässigt die Menschenrechte. Beide Vorstellungen sind weitgehend antagonistisch.

Die Psychoanalyse ist ein wunderbares Instrument, um, mehr oder weniger in der ganzen Welt, die Menschen besser zu verstehen. Ich glaube aber, dass es ein Fehler wäre, es ohne Anpassung an die verschiedenen Kulturen anzuwenden. Die Entwicklungsphasen des Menschen - auch wenn sie hinsichtlich ihrer biologischen Gesetzmässigkeiten gleich sind - haben unterschiedliche Aufgaben und werden unterschiedlich behandelt. Die Abwehr- und Anpassungsmechanismen gehorchen kulturvermittelten Regeln und Wertvorstellungen. Und offensichtlich muss man auch Therapieziele unterschiedlich definieren. Die kulturelle Komplexität zu 
verleugnen, wäre ein grosser Fehler. Es ist Zeit für eine umfassende transkulturelle Debatte.

Zum Schluss möchte ich noch einmal an den Anfang zurückzukehren, dem ersten Kultur- und Wissenschaftsaustausch zwischen Ost und West durch die Jesuiten, der in dem Briefwechsel mit Leibniz so aufregend nachzulesen ist. Es war ein Wissensaustausch ohne Beispiel, in dem Leibniz seine Wissenschaft als Mission empfand und selbst begeistert war von dem, was er über China lernte. Wichtig war, dass die sprachkundigen Jesuiten sich den Sitten und Gepflogenheiten der chinesischen Elite anpassten. sie trugen die Seidengewänder der chinesischen Literaten und verstanden sich als «Gelehrte aus dem Westen». Es kam also zu einer «Akkommodation» an die vorgefundenen Verhältnisse. Ende des 17. Jahrhunderts waren 300000 Chinesen zum Christentum konvertiert. Im Jahr 1692 erliess der grosse Philosophenkaiser Kang Xi, der die Arbeit der Patres unterstützte, ein Toleranzedikt, das den chinesischen Christen die freie Ausübung ihrer Religion gestattete (Albrecht, 1985). Der Erfolg der Jesuiten lag unter anderem darin, dass sie den Konvertiten nicht versagten, die staatlich vorgeschriebenen Gebräuche wie den Konfuzius- und Ahnenkult weiterauszuüben, weil diese, ihrer Meinung nach, mit dem Christentum kompatibel waren. Es kam dann aber zu einem von Teilen der katholischen Kirche, insbesondere auch von anderen Orden, angezettelten «Ritenstreit». Die traditionellen chinesischen Sitten wurden als «Götzendienst» verurteilt. Im Jahr 1715 verbot dann schliesslich der Papst die China-Mission der Jesuiten, und ein einzigartiger Brückenschlag zwischen beiden Kulturen wurde für lange Zeit unterbrochen.

So gewagt es ist, die Einführung des Christentums mit der der Psychoanalyse zu vergleichen: Ich glaube, dass das Beispiel lehrreich ist und die Frage aufwirft, inwieweit eine «Akkommodation» der Psychoanalyse an chinesische Verhältnisse denkbar ist. Möglicherweise steht ein «Ritenstreit» noch bevor.

\section{Literatur}

Albrecht, M. (Hrsg.). (1985). Christian Wolff: Rede über die praktische Philosophie der Chinesen. Einleitung. Mehnert: Hamburg.

Basch M.F. (1983). Empathic understanding. J Am Psychoanal Assoc, 31, 101-124. Bell, D. (2009). The confucian party. New York Times, 15.12.2009.

Benedict, R. (1946). The chrysanthemum and the sword: patterns of Japanese culture. Mifflin: Boston.

Blowers, G. (2006). Crossing borders: oedipus in Asia and the resistance to psychoanalysis. China Cross Curr, 3, 8-27. 
Bond, M.H. \& Hwang, K. K. (1986). The social psychology of the chinese people. In Bond, M.H. (Hrsg.), The psychology of the chinese people (S. 213-266). Oxford: Oxford University Press.

Buschmann, S. (2009). Leibniz und china:Vor 300 Jahren geschrieben - heute hochaktuell. www.chinato-day.com.cn/ctgerman/buk/txt/2009-08. Zugegriffen: 9. Mai 2011.

Cheung, F., Gan, Y. \& Lo, P. (2005). Personality and psychopathology: insights from chinese studies. In Tseng, W. S., Chang, S. C., Nishizono, M. (Hrsg.), Asian culture and psychotherapy (S. 21-39). Honolulu: University of Hawai'i Press.

Ching, M. L. (1997). A cultural perspective on the study of chinese adolescent development. Child Adolesc soc Work J, 14, 95-113.

Creighton, M. R. (1990). Revisiting shame and guilt cultures: a forty-year pilgrimage. Ethos, 18, 279-307.

English, T. \& Chen S. (2007). Culture and self concept stability: consistency across and within contexts among Asian Americans and European Americans. J Pers soc Psychol, 93, 478-490.

Fuligni, A. \& Zhang, W. (2004). Attitudes toward family obligation among adolescents in contemporary urban and rural China. Child Dev, 75, 180-192.

Gerlach, A. (2010). Grossgruppenidentität und psychosoziale Abwehr. Forum Psychoanal, 26, 241-254.

Giddens, A. (2001). Entfesselte Welt. Wie die Globalisierung unser Leben verändert. Frankfurt a. M.: Suhrkamp.

Haag, A. (2011). Versuch über die moderne Seele Chinas. Gießen: Psychosozial.

Haag, A. \& Zhao, M. (2004). Kollektive Traumatisierung - chinesische Schicksale im 20. Jahrhundert. Psyche - Z Psychoanal, 58, 352-366.

Haaß-Wiesegart, M. \& Schweitzer, J. (2004). Psychotherapie-Ausbildung in China. Psychother Dialog, 5, 407-413.

Hall, E. T. (1989). Beyond culture. New York: Anchor.

Heine, S. J. (2001). Self as cultural product: an examination of East Asian and north American selves. J Pers, 69, 881-906.

Hua, W., Beitel, M., Schuman-Olivier, Z. \& Barry, D. (2007). Psychometric properties of a chinese version of the psychological mindedness scale. JAm Psychoanal Assoc, 55, 300-305.

Huntington, S., New York, P. (1998). Kampf der Kulturen. Berlin: Siedler.

Johnson, F. (1985). The western concept of self. In Marseille, A. J., Devos, G. \& Hsu L. K. S. (Hrsg.), Culture and self(S. 91-138). London: Tavistock. 
Jullien, F. (2006). Vortrag vor Managern über Wirksamkeit und Effizienz in China und im Westen. Berlin: Merve.

Kakar, S. (1985). Psychoanalysis in non-western cultures. Int rev Psychoanal, 12, 441-448.

Leibniz, G.W. (2006). Der Briefwechsel mit den Jesuiten in China (1689-1714). In Widmayer, R. (Hrsg.). Hamburg: Meiner.

Lu, X. (1994). Die wahre Geschichte des Herrn Jedermann. In Lu, X. (Hrsg.), Werke in sechs Bänden, Bd. 1 (S. 104-164). Zürich: Unionsverlag.

Markus, H. R. \& Kitayama, S. (1991). Culture and the self: implications for cognition, emotion, and motivation. Psychol Rev, 98, 224-253.

Markus, H. R. \& Kitayama, S. (1998). The cultural psychology of personality. J Cross Cult Psychol, 29, 63-87.

Ng, M.L. (1985). Psychoanalysis for the chinese - applicable or not applicable? Int Rev Psychoanal, 12, 449-460.

Nisbett, R.E. (2003). The geography of thought. How Asians and Westerners think differently... and why. New York: Free Press.

Simon, F., Haass-Wiesegart, M. \& Zhao X. (2011.) «Zhong De Ban» oder: Wie die Psychotherapie nach China kam. Heidelberg: Auer.

Tam, K. K. (1995). Self-identity and the problematic of chinese modernity. "The humanities". Bulletin, Nr. 4, 57-64.

Triandis, H.C., Bontempe, R. \& Villareal, M. J. (1988). Individualism and collectivism: cross-cultural perspectives on self-ingroup relationships. J Personal Soc Psychol, 54, 323-338.

Tseng, W.S. (1995). Psychotherapy for the chinese: cultural adjustments. In Cheng, Y.C., Baxter, H. \& Cheung, F. (Hrsg.), Psychotherapy for the chinese II. Department of Psychiatry (S. 1-22). The Chinese University of Hongkong.

Tseng, W. S. (2005). Integration and application for therapy. In Tseng, W. S., Suk, C. C. \& Nishizono, M. (Hrsg.), Asian culture and psychotherapy (S. 265-279). Honolulu: University of Hawai'i Press.

Tung, M. (1991). Insight - oriented psychotherapy and the chinese patient. Am J Orthopsychiatr, 61, 186-194.

Xiao, Z. (2008). Application of psychoanalytically oriented therapy for the chinese: cultural considerations. World Cult Psychiatr Res Rev, 3, 20-23.

Zeng, Y. (1991). Family dynamics in china. Wisconsin: University of Wisconsin Press.

Zhang, T. (2007) Die chan-buddhistische Praxis des Can Huatou und ihre Anwendung in der Psychotherapie. Vortrag auf dem Kongress "Changing society- 
changing people-psychotherapeutic answers" in Shanghai 20.-23. Mai 2007. Übers. v. H. Schultz unveröffentlicht.

Zhao, X. (2002). Die Einführung systemischer Familientherapie in China als kulturelles Projekt. Berlin: VWB.

Zhao, X. (2009). Mental health in contemporary China. In Incayawar, M., Bouchard, L. \& Wintrob, R. (Hrsg.), Psychiatrist and traditional healers (S. 135-148). Chichester: Wiley.

\section{Anmerkung}

1 Online publiziert: 24. April 2012 @ Springer-Verlag 2012. 